Pacific

Journal of

Mathematics

SINGULAR ANGLES OF WEAK LIMITING METRICS UNDER CERTAIN INTEGRAL CURVATURE BOUNDS

Qing Chen, Xiuxiong Chen and Weiyong He 


\title{
SINGULAR ANGLES OF WEAK LIMITING METRICS UNDER CERTAIN INTEGRAL CURVATURE BOUNDS
}

\author{
Qing Chen, Xiuxiong Chen and Weiyong He
}

\begin{abstract}
We prove that a nonvanishing weak limit of Riemannian metrics in surfaces with an integral curvature bound admits only weak cusp singularities. The result is useful toward generalizing classical uniformization theory to surfaces with boundary.
\end{abstract}

\section{Introduction}

Classical uniformization theorem says that in a compact Riemann surface without boundary there is a constant curvature metric in any conformal class of metrics. There have been many attempts to generalize this theory to surfaces with boundary. The main focus, started by the independent work of Troyanov [1991] and McOwen [1988], has been to study the existence or nonexistence of constant curvature metrics in surfaces with conical singularities. Much work has followed since; see, for example, [Chen and Li 1991; Chang and Yang 1988; Luo and Tian 1992].

The disadvantage of this classical approach is that the Gaussian curvature is a second-order differential operator of the metric, while the condition of prescribing conical singularities is equivalent to prescribing both the metric and its derivatives near the set of singular points at infinitesimal level. Thus, the constant curvature equation with prescribed conical singularities is an overdetermined elliptic equation. In general, one should not expect to get a clear-cut statement about the existence (or nonexistence) of solutions.

We now describe another approach. Given a compact Riemann surface $\Omega$ and a Hermitian metric $g_{0}$ on $\Omega$, any metric $g$ on $\Omega$ is said to be conformal to $g_{0}$ if there exists a smooth function $e^{2 \varphi}$ such that $g=e^{2 \varphi} g_{0}$ on $\Omega$. Define the variational space $\mathscr{G}(\Omega)$ to be the set of all metrics that are conformal to $g_{0}$ and agree with $g_{0}$ on $\partial \Omega$ up to first derivatives. For each $g \in \mathscr{G}(\Omega)$, define the energy functional

$$
E(g)=\int_{\Omega} K^{2} d g
$$

MSC2000: primary 53C20; secondary 53C23.

Keywords: Riemannian metric, weak limit, singular angle.

Qing Chen was supported by the NCFT Program of the Ministry of Education of China. Xiuxiong Chen was partially supported by an NSF grant. 
where $K$ is the Gaussian curvature of $g$. The problem is to minimize this functional with the area constraint

$$
A(g)=\int_{\Omega} d g=\text { const. }
$$

A critical point of this functional is called an extremal Hermitian metric; see [Calabi 1982]. The Euler-Lagrange equation is

$$
\triangle_{g} K+K^{2}=C
$$

where we have, in a local system,

$$
-\triangle \varphi=K e^{2 \varphi} \quad \text { if } g=e^{2 \varphi}|d z|^{2} .
$$

By a theorem of Calabi, any metric solving this Euler-Lagrange equation on a surface without boundary must have constant scalar curvature. This result conforms with the classical uniformization theorem.

One of us (X. Chen) has been using the approach just described, namely the study of the variational problem of minimizing $E(g)$ in $\mathscr{G}(\Omega)$ with fixed area, with the goal of generalizing uniformization results to surfaces with boundary. Some of the ideas go back to E. Calabi (private communications).

To attack this variational problem, one first studies the weak compactness of any subset of metrics in $\mathscr{G}(\Omega)$ with finite energy and area. Generally speaking, for a sequence of metrics $\left\{g_{n}, n \in \mathbb{N}\right\}$ with finite energy and area, there exists a weak limit metric $g$ such that $g_{n}$ converges to $g$ weakly in any compact subset away from a finite set of singular points. An important feature of the limit is the bubbling phenomenon, first observed by Sacks and Uhlenbeck in 1979, when they studied the existence theorem for harmonic maps between two spheres [Uhlenbeck 1982]. The key observation was that the noncompactness is associated with the concentration of the energy density at isolated bubble points. Around each such point, one can define a weak singular angle in the approximation sense (see Definition 2 below). If the weak singular angle is 0 , it is called a weak cusp singular point. An intriguing question is that whether all points is weak cusp singular points if the weak limit metric $g \neq 0$. In this paper, we will prove (see Section 2 for terminology and notation):

Theorem 1.1. Let $\left\{g_{n}\right\}$ be a sequence of conformal metrics in domain $\Omega$ with finite area and finite energy. There exist a subsequence of $\left\{g_{n}\right\}$, a limit metric $g_{0}$ and a finite set of bubble points $\left\{p_{1}, p_{2}, \ldots, p_{m}\right\}$ such that $g_{n} \rightarrow g_{0}$ in $H_{\mathrm{loc}}^{2,2}\left(\Omega \backslash\left\{p_{1}, p_{2}, \ldots, p_{m}\right\}\right)$. If $g_{0} \neq 0\left(\varphi_{0} \neq-\infty\right)$, then $g_{0}$ has a weak cusp singularity at each bubble point $p_{i}$. There is no ghost vertex in the bubble-tree decomposition.

This motivates the following classification result: 
Theorem 1.2 [Chen 1999]. Let $\Omega$ be a surface without boundary and let $g$ be an extremal Hermitian metric in $\Omega \backslash\left\{p_{1}, \ldots, p_{n}\right\}$ with finite energy and area, and having only weak cusp singularities at all singular points.

(1) If $\chi(\Omega) \leqslant 0$, then $K$ is a negative constant.

(2) If $n \geqslant 3$ and $\chi(\Omega)=2$ (that is, $\Omega$ is a sphere), then $K$ is a negative constant.

(3) If $n=2$ and $\chi(\Omega)=2$, there exists no extremal Hermitian metric.

(4) If $n=1$ and $\chi(\Omega)=2$, there exists a unique extremal Hermitian metric determined by total area, and the metric must be rotationally symmetric.

These results are critical for the generalization of uniformization theory to a surface with boundary.

The main result was stated in [Chen 1998a; 1999] without proof and was used crucially in [Chen 2001], in deriving the long-time existence of the Calabi flow in Riemannian surfaces. At present, there is strong interest in the Calabi flow for general Kähler manifolds. Clarification of this important technical step will likely be indicative of what happens in more general settings.

\section{The problem from an analytic viewpoint}

In a coordinate chart $(D, z)$, any metric $g$ can be written as

$$
g=e^{2 \varphi}\left(d x^{2}+d y^{2}\right)
$$

and the curvature function is

$$
K=-\frac{\triangle \varphi}{e^{2 \varphi}} .
$$

A metric is said to have finite area $C_{1}$ and finite energy $C_{2}$ if

$$
\int_{D} e^{2 \varphi} d x d y \leqslant C_{1} \text { and } \int_{D} \frac{(\triangle \varphi)^{2}}{e^{2 \varphi}} d x d y \leqslant C_{2}
$$

A sequence of metrics $\left\{g_{n}\right\}$, where $g_{n}=e^{2 \varphi_{n}}\left(d x^{2}+d y^{2}\right)$, is said to have finite area $C_{1}$ and finite energy $C_{2}$ if each $\varphi_{n}$ satisfies (2-1). From now on we will always use either $\left\{\varphi_{n}\right\}$ or $\left\{g_{n}\right\}$ to denote a sequence of metrics with finite area and finite energy.

For any subdomain $\Omega$ in $D$, relabel the energy and area for a conformal parameter function as

$$
A_{c}(\varphi, \Omega)=\int_{\Omega} e^{2 \varphi} d x d y, \quad K_{c}(\varphi, \Omega)=\int_{\Omega} \frac{(\triangle \varphi)^{2}}{e^{2 \varphi}} d x d y .
$$

A "zero metric" should have zero area and zero energy. Since the zero metric has a conformal parameter function identically equal to $-\infty$, we define $A_{c}(-\infty, \Omega)=$ $K_{c}(-\infty, \Omega)=0$. For notational convenience, define $\hat{H}_{\mathrm{loc}}^{2,2}(\Omega)=H^{2,2}(\Omega) \cup\{-\infty\}$. 
A sequence of functions $\left\{\varphi_{n}\right\} \in H^{2,2}(\Omega)$ is said to converge weakly to a function $\varphi_{0}$ in $\hat{H}_{\mathrm{loc}}^{2,2}(\Omega)$ if one of two mutually exclusive alternatives holds:

1 (Vanishing case): If $\varphi_{0} \equiv-\infty$, then $\varphi_{n} \rightarrow-\infty$ uniformly in any relatively compact subdomain of $\Omega$.

2 (Nonvanishing case): If $\varphi_{0} \in H^{2,2}(\Omega)$, then $\varphi_{n} \rightarrow \varphi_{0}$ weakly in $H_{\text {loc }}^{2,2}(\Omega)$.

Consequently, a sequence of Riemannian metrics $\left\{g_{n}\right\} \in H^{2,2}(\Omega)$ converges weakly to a limit metric $g_{0}$ in $H_{\mathrm{loc}}^{2,2}(\Omega)$ if and only if either

$1 g_{n} \rightarrow 0$ everywhere (and $g_{0} \equiv 0$ ), or

$2 \varphi_{n} \rightarrow \varphi_{0}$ in $H_{\mathrm{loc}}^{2,2}(\Omega)$, where $g_{n}=e^{2 \varphi_{n}} g_{\mathrm{bk}}$ and $g_{0}=e^{2 \varphi_{0}} g_{\mathrm{bk}}$, for $g_{\mathrm{bk}}$ a smooth background metric in $\Omega$.

Definition 2.1. A point $p$ is said to be a bubble point of $\left\{\varphi_{n}\right\}$ if, for any $r>0$,

$$
\liminf _{n \rightarrow \infty} \int_{D_{r}(p)} \frac{\triangle\left(\varphi_{n}\right)^{2}}{e^{2 \varphi_{n}}} d x d y \geqslant \alpha>0 \quad \text { and } \quad \liminf _{n \rightarrow \infty} \int_{D_{r}(p)} e^{2 \varphi_{n}} d x d y \geqslant \beta>0,
$$

where $D_{r}(p)$ is a coordinate disk centered at $p$ with radius $r$.

The largest possible numbers $\alpha$ and $\beta$ are the concentration weights of the energy function and area function at point $p$.

For convenience we restate here the three main theorems from [Chen 1998b]. Their proofs can be found there.

Theorem 2.2. Let $\left\{\varphi_{n}, n \in \mathbb{N}\right\}$ be a sequence of metrics in $H^{2,2}(D)$ with finite area $C_{1}$ and energy $C_{2}$. There exists a subsequence $\left\{\varphi_{n_{j}}, j \in \mathbb{N}\right\}$ of $\left\{\varphi_{n}\right\}$, a finite number of bubble points $\left\{p_{1}, p_{2}, \ldots, p_{m}\right\}$ with respect to $\left\{\varphi_{n_{j}}, j \in \mathbb{N}\right\}$ (where $\left.0 \leqslant m \leqslant \sqrt{C_{1} C_{2} /(4 \pi)}\right)$, and a metric $\varphi_{0} \in \hat{H}_{\mathrm{loc}}^{2,2}\left(D \backslash\left\{p_{1}, p_{2}, \ldots, p_{m}\right\}\right)$ such that

$$
\varphi_{n_{j}} \rightarrow \varphi_{0} \quad \text { in } \hat{H}_{\mathrm{loc}}^{2,2}\left(D \backslash\left\{p_{1}, p_{2}, \ldots, p_{m}\right\}\right) .
$$

Theorem 2.3 (Bubbles on bubbles). Let $\left\{\varphi_{n}\right\}$ be a sequence of metrics in D with finite area $C_{1}$ and finite energy $C_{2}$. Suppose that $p=0$ is the only bubble point in $D$, that it has area concentration $A_{p}$ and energy concentration $K_{p}$, and that there exists a metric $\varphi_{0} \in \hat{H}_{2,2}(D \backslash\{p\})$ such that $\varphi_{n} \rightarrow \varphi_{0}$ in $\hat{H}_{2,2}(D \backslash\{p\})$. A sequence of numbers $\left\{\delta_{n} \searrow 0\right\}$ can be chosen to renormalize the sequence of metrics as $\phi_{n}(x, y)=\varphi_{n}\left(\delta_{n} x, \delta_{n} y\right)+\log \delta_{n}$, for $n \in \mathbb{N}$.

There exists a subsequence $\left\{\varphi_{n_{j}}, j \in \mathbb{N}\right\}$ of $\left\{\varphi_{n}\right\}$, a finite number of bubble points $\left\{q_{1}, q_{2}, \ldots, q_{m}\right\}$ with respect to the subsequence of metrics $\left\{\phi_{n_{j}}\right\}$ (where $0 \leqslant m \leqslant$ $\left.\sqrt{A_{p} K_{p} /\left(4 \pi^{2}\right)}\right)$, and a metric $\phi_{0} \in \hat{H}^{2,2}\left(S^{2} \backslash\left\{\infty, q_{1}, q_{2}, \ldots, q_{m}\right\}\right)$ such that

$$
\phi_{n_{j}} \rightarrow \phi_{0} \in \hat{H}_{\mathrm{loc}}^{2,2}\left(S^{2} \backslash\left\{\infty, q_{1}, q_{2}, \ldots, q_{m}\right\}\right) .
$$

If $\phi_{0} \equiv-\infty$ (vanishing case), then $m \geqslant 2$ and $p$ is a bubble point of $\left\{\phi_{n_{j}}, j \in \mathbb{N}\right\}$. 
Theorem 2.4 (Bubble tree). The limit of any locally weakly convergent sequence of metrics $\left\{g_{k}, k \in \mathbb{N}\right\}$ in $\mathscr{G}(\Omega)$ encompasses the following data:

(1) A finite rooted tree $T$, possibly reduced to just the base vertex $f$.

(2) The base vertex $f \in T$ is a limit metric in $\Omega$ with a finite number of bubble points $\left\{p_{i}\right\}$ deleted; the edge emanating from the base vertex is $\left\{p_{i}\right\}$.

(3) Any other vertex $f_{s}$ is a limit metric defined on $S^{2} \backslash\left\{\infty, p_{s_{i}}\right\}$; the edges emanating from this vertices are $\left\{p_{s_{i}}\right\}$.

(4) For each pair of vertex $f_{s_{1}}$ and $f_{s_{2}}$ bounding a common edge in $T$, they are tenuously connected at the pair of respective singular points. The number of vertices whose valence $\neq 2$, is bounded from above by $\sqrt{C_{1} C_{2}}$. The depth of the tree is also finite in a reasonable sense. Each vertex $f_{I}=f_{i_{1} i_{2} \cdots i_{k}}$ has the property that, if it vanishes in any point in its domain, it vanishes everywhere; in this case we call it a ghost vertex. The number of ghost vertices is finite.

Definition 2.5. Around each bubble point, define the weak singular angle

$$
\alpha=\lim _{r \rightarrow 0} \int_{\partial D_{r}(p)} k_{g} d s_{g}=\lim _{r \rightarrow 0} \lim _{n \rightarrow \infty} \int_{\partial D_{r}(p)} k_{g_{n}} d s_{g_{n}}
$$

if the limit is well defined. Here $k_{g}$ and $k_{g_{n}}$ are geodesic curvatures of $g$ and $g_{n}$.

The weak angle does exist, as we shall see in the next section, and has some interesting properties. From now on, we will always assume that $\left\{g_{n}\right\}$ converges, by substituting a convergent subsequence if necessary.

If the weak singular angle is zero, the bubble point $p$ is called a weak cusp singularity. Our purpose is to prove:

Main Theorem 2.6. Let $\left\{g_{n}\right\}$ be a sequence of conformal metrics in $\Omega$ with finite area and finite energy. There exists a subsequence of $\left\{g_{n}\right\}$, a limit metric $g_{0}$ and a finite set of bubble points $\left\{p_{1}, p_{2}, \ldots, p_{m}\right\}$ such that $g_{n} \rightarrow g_{0}$ in $H_{\mathrm{loc}}^{2,2}\left(\Omega \backslash\left\{p_{1}, p_{2}, \ldots, p_{m}\right\}\right)$ (following the notations of Theorem 1.1). If $g_{0} \neq 0$, then $g_{0}$ has a weak cusp singularity at each bubble point $p_{i}$, and there is no ghost vertex in the bubble-tree decomposition.

\section{Asymptotic geometry of singular points}

In this section, we introduce the blowing-up process and the definition of a neck. We also prove several lemmas that are essential to our main theorem.

Definition 3.1. For any metric $\varphi$ with finite area $C_{1}$ and energy $C_{2}$ in $D \backslash\{0\}$, set

$$
\bar{\varphi}(r)=\frac{1}{2 \pi} \int_{0}^{2 \pi} \varphi(r \cos \theta, r \sin \theta) d \theta .
$$


Lemma 3.2 [Chen 1998b]. Suppose $\varphi$ is a metric with finite area $C_{1}$ and energy $C_{2}$ in $D \backslash\{0\}$.

(1) $\lim _{r \rightarrow 0}(\varphi(r \cos \theta, r \sin \theta)+\log r)=-\infty$.

(2) $\lim _{r \rightarrow 0} \bar{\varphi}_{r}(r) r$ exists and is finite.

(3) There exists constants $\lambda \in(0,1)$ and $C_{3}, C_{4}$ such that

$$
\frac{1}{\lambda}(\bar{\varphi}(r)+\log r)+C_{3} \leq \varphi(r \cos \theta, r \sin \theta)+\log r \leq \lambda(\bar{\varphi}(r)+\log r)+C_{4} .
$$

Similarly, one can prove:

Corollary 3.3. Suppose $\phi$ is a metric in $R^{2} \backslash \bar{D}_{r_{0}}(0)$ with finite area $C_{1}$ and energy $C_{2}$, where $\bar{D}_{r_{0}}(0)$ is a closed disk with radius $r_{0}$.

(1) $\lim _{r \rightarrow \infty}(\phi(r \cos \theta, r \sin \theta)+\log r)=-\infty$.

(2) $\lim _{r \rightarrow \infty} \bar{\phi}_{r}(r) r$ exists and is finite.

(3) There exists constants $\mu \in(0,1)$ and $C_{5}, C_{6}$ such that

$$
\frac{1}{\mu}(\bar{\varphi}(r)+\log r)+C_{5} \leq \varphi(r \cos \theta, r \sin \theta)+\log r \leq \mu(\bar{\varphi}(r)+\log r)+C_{6} .
$$

Lemma 3.4 [Chen 1998b]. Let $\left\{\varphi_{n}\right\}$ be a sequence of metrics with finite area $C_{1}$ and energy $C_{2}$. There exists a constant $\epsilon_{0}$ such that if

$$
\max _{r \leqslant \rho}\left|\partial D_{r}\right|_{g_{n}}=\max _{r \leqslant \rho} \int_{0}^{2 \pi} e^{\varphi_{n}(r \cos \theta, r \sin \theta)} r d \theta \leqslant \epsilon_{0} \quad \text { for all } n \in \mathbb{N},
$$

then $\left\{\varphi_{n}\right\}$ has no bubble points in $D$.

We can now explain the blowing-up process. To simplify the problem, let $\left\{g_{n}=\right.$ $\left.e^{\varphi_{n}}\left(d x^{2}+d y^{2}\right)\right\}$ be a sequence of metrics in $D$ with finite area and finite energy, converging to a limit metric $g=e^{\varphi_{0}}\left(d x^{2}+d y^{2}\right) \neq 0$, and having $\{0\}$ as its only bubble point. Following Lemma 3.2, we have

$$
\lim _{r \rightarrow 0} \max _{0 \leqslant \theta \leqslant 2 \pi}\left(\varphi_{0}(r \cos \theta, r \sin \theta)+\log r\right)=-\infty .
$$

Then there exists $r_{1}>0$ such that

$$
\max _{0 \leqslant \theta \leqslant 2 \pi}\left(\varphi_{0}(r \cos \theta, r \sin \theta)+\log r\right) \ll 0 \quad \text { for all } r \leq r_{1} .
$$

If $n$ is large enough, the convergence implies that

$$
\max _{0 \leqslant \theta \leqslant 2 \pi}\left(\varphi_{n}\left(r_{1} \cos \theta, r_{1} \sin \theta\right)+\log r_{1}\right) \ll 0 \quad \text { for all } n>N
$$


equivalently, if the length of the circle $|z|=r_{1}$ is very small, then

$$
\left|\partial D_{r_{1}}\right|_{g_{n}}=\int_{0}^{2 \pi} e^{\varphi_{n}\left(r_{1} \cos \theta, r_{1} \sin \theta\right)} r_{1} d \theta \leq \varepsilon \quad \text { for all } n>N .
$$

According to Lemma 3.4, if $\varepsilon$ is small enough, for each $n>N$ we can choose $\delta_{n}$ such that

$$
\left|\partial D_{r}\right|_{g_{n}}=\int_{0}^{2 \pi} e^{\varphi_{n}(r \cos \theta, r \sin \theta)} r d \theta<\varepsilon \quad \text { for all } r \text { with } r_{1} \geq r>\delta_{n},
$$

and

$$
\left|\partial D_{\delta_{n}}\right|_{g_{n}}=\int_{0}^{2 \pi} e^{\varphi_{n}\left(\delta_{n} \cos \theta, \delta_{n} \sin \theta\right)} \delta_{n} d \theta=\varepsilon .
$$

Renormalize this sequence of metrics as

and

$$
\phi_{n}(z)=\varphi\left(\delta_{n} z\right)+\log \delta_{n} \quad \text { for all }|z|<\frac{1}{\delta_{n}}
$$

$$
\tilde{g}_{n}(z)=e^{2 \phi_{n}(z)}|d z|^{2}=g_{n}\left(\delta_{n} z\right) .
$$

The theorems in Section 2 assert that there is a subsequence $\left\{\tilde{g}_{n_{j}}\right\}$ of metrics $\left\{\tilde{g}_{n}\right\}$, a limit metric $\tilde{g}$ and finitely many bubble points $\left\{q_{1}, q_{2}, \ldots, q_{m}\right\}$ such that either

$$
\tilde{g} \equiv 0\left(\phi_{n_{j}} \rightarrow-\infty\right) \quad \text { or } \quad \tilde{g}_{n_{j}} \rightarrow \tilde{g}
$$

in $\hat{H}_{\mathrm{loc}}^{2,2}\left(S^{2} \backslash\left\{\infty, q_{1}, q_{2}, \ldots, q_{m}\right\}\right)$.

Choose $r_{2}$ big enough that $\left\{q_{1}, q_{2}, \ldots, q_{m}\right\} \subset D_{r_{2}}$. The annulus bounded by the two circles $|z|=r_{1}$ and $|z|=r_{2} \delta_{n}$ is called the neck of the blowing-up process and denoted by $\operatorname{Neck}\left(r_{1}, r_{2}\right)$. The blowing up procedure or the renormalization procedure depends only on the filter size $\varepsilon>0$ (once a coordinate system is fixed). Since $g_{n} \rightarrow g_{0}$ in $\hat{H}_{\text {loc }}^{2,2}(D \backslash\{p\})$, the surface $\left(g_{0}, D \backslash\{p\}\right)$ and $\left(\tilde{g}_{0}, S^{2} \backslash\left\{\infty, q_{1}, q_{2}, \ldots, q_{m}\right\}\right)$ are called tenuously connected at $p$ and at $z=\infty$.

Lemma 3.5. If the limit metric $g$ is nonzero, the weak angle $\alpha$ exists and is finite, and $\alpha \geqslant 0$.

Proof. In polar coordinates $(r, \theta)$, we have

$\alpha=\lim _{r \rightarrow 0} \int_{\partial D_{r}(p)} k_{g} d s_{g}=\frac{1}{2 \pi} \lim _{r \rightarrow 0} \int_{0}^{2 \pi}\left(\partial_{r} \varphi(r \cos \theta, r \sin \theta) r+1\right) d \theta=\lim _{r \rightarrow 0} \bar{\varphi}^{\prime}(r)+1$.

According to Lemma 3.2, the weak angle exists and is finite. Now we only have to prove $\alpha \geq 0$. Assume $\alpha<0$. By the preceding equality we know that $\bar{\varphi}_{r}(r) r+1<0$ for $r$ small enough, say for $r<r_{0}$; that is,

$$
\bar{\varphi}_{r}(r)+\frac{1}{r}<0 \quad \text { when } r<r_{0} .
$$


Then, for $0<r_{1}<r_{2}<r_{0}$,

$$
0>\int_{r_{1}}^{r_{2}}\left(\bar{\varphi}_{r}(r)+\frac{1}{r}\right)=\left.(\bar{\varphi}(r)+\log r)\right|_{r 1} ^{r_{2}}=\left(\bar{\varphi}\left(r_{2}\right)+\log r_{2}\right)-\left(\bar{\varphi}\left(r_{1}\right)+\log r_{1}\right) .
$$

Letting $r_{1} \rightarrow 0$, since $\bar{\varphi}\left(r_{1}\right)+\log r_{1} \rightarrow-\infty$, it follows that

$$
0>\int_{r_{1}}^{r_{2}}\left(\bar{\varphi}_{r}(r)+\frac{1}{r}\right)=\left(\bar{\varphi}\left(r_{2}\right)+\log r_{2}\right)-\left(\bar{\varphi}\left(r_{1}\right)+\log r_{1}\right) \rightarrow \infty,
$$

which is a contradiction.

Definition 3.6. Let $\left\{\varphi_{n}\right\}$ be a sequence of metrics in $D$ with finite area $C_{1}$ and finite energy $C_{2}$. Suppose that $p=0$ is the only bubble point in $D$ in the blowingup process just described in Lemma 3.4. We define the out angle $\beta$ around $p$ in $\operatorname{Neck}\left(r_{1}, r_{2}\right)$ as

$$
\beta=\lim _{r_{2} \rightarrow \infty} \lim _{n \rightarrow \infty} \int_{\partial D_{r_{2} \delta_{n}}(p)} k_{g_{n}} d s_{n}
$$

if the limit exists, where $k_{g_{n}}$ is the geodesic curvature.

Lemma 3.7. In the notations in Lemma 3.4, if the (renormalized) limit metric $\phi_{0}$ is not $-\infty$, the out angle $\beta$ exists and is finite. Furthermore, $\beta \leqslant 0$.

Proof. By the definition of $\beta$, we have

$$
\beta=\lim _{r_{2} \rightarrow \infty} \lim _{n \rightarrow \infty} \int_{\partial D_{r_{2} \delta_{n}}(p)} k_{g_{n}} d s_{n}=\lim _{r_{2} \rightarrow \infty} \lim _{n \rightarrow \infty} \int_{\partial D_{r_{2}}(p)} k_{\tilde{g}_{n}} d \tilde{s}_{n} .
$$

If the limit metric $\tilde{g}_{0}$ does not vanish, that is, if $\phi_{0} \neq-\infty$, we have

$$
\beta=-\lim _{r_{2} \rightarrow \infty} \int_{\partial D_{r_{2}}} k_{\tilde{g}_{0}} d \tilde{s}_{0} .
$$

By Lemma 3.2, $\beta$ is well defined and finite. Just as the argument in the proof of Lemma 3.7, we can then prove that $\beta \leqslant 0$.

Remark 3.8. The out angle $\beta$ (more precisely, its negative) can be regarded as the weak angle at $\{\infty\}$ in the blowing-up process.

When the limit metric vanishes, the weak angle can still be defined:

Lemma 3.9. If a sequence of metrics $\left\{g_{n}=e^{\varphi_{n}}\left(d x^{2}+d y^{2}\right)\right\}$ on D has vanishing limit metric and $\{0\}$ as its only bubble point, the weak angle at $\{0\}$ exists and finite.

Proof. It suffices to show the existence of the limit

$$
\alpha=\lim _{r \rightarrow 0} \lim _{n \rightarrow \infty} \int_{\partial D_{r}(p)} k_{g_{n}} d s_{g_{n}}=\lim _{r \rightarrow 0} \lim _{n \rightarrow \infty} \int_{0}^{2 \pi}\left(\partial_{r} \varphi_{n} r+1\right) d \theta .
$$


But for any $0<r_{1}<r_{2}$,

$$
\begin{aligned}
\mid \int_{0}^{2 \pi}\left(\partial_{r} \varphi_{n}\left(r_{2} \cos \theta, r_{2} \sin \theta\right) r_{2}\right. & +1) d \theta-\int_{0}^{2 \pi}\left(\partial_{r} \varphi_{n}\left(r_{1} \cos \theta, r_{1} \sin \theta\right) r_{1}+1\right) d \theta \mid \\
& =\int_{r_{1}}^{r_{2}} \int_{0}^{2 \pi} \partial_{r}\left(\partial_{r} \varphi_{n} r\right) d \theta d r=\int_{r_{1}}^{r_{2}} \int_{0}^{2 \pi} \Delta \varphi_{n} d \theta d r \\
& \leqslant \int_{r_{1}}^{r_{2}} \int_{0}^{2 \pi} \frac{\left(\triangle \varphi_{n}\right)^{2}}{e^{2 \varphi_{n}}} d \theta d r \int_{r_{1}}^{r_{2}} \int_{0}^{2 \pi} e^{2 \varphi_{n}} d \theta d r \\
& =K_{n}\left(r_{1}, r_{2}\right) A_{n}\left(r_{1}, r_{2}\right)
\end{aligned}
$$

which tends to 0 as $n \rightarrow \infty$. Hence the limit exists and $\alpha$ is defined.

\section{Proof of the main theorem}

In the neck of the blowing-up process (page 41), the sequence of metrics has an average property around concentric circles:

Lemma 4.1 (Max-min inequality). Let $\left\{g_{n}=e^{2 \varphi_{n}}\left(d x^{2}+d y^{2}\right)\right\}$ be a sequence of metrics with finite area and finite energy, converging weakly to a limit metric $g=e^{2 \varphi}\left(d x^{2}+d y^{2}\right)$ in $H_{\mathrm{loc}}^{2,2}(D \backslash\{0\})$ and having $\{0\}$ as its only bubble point. In $\operatorname{Neck}\left(r_{1}, r_{2}\right)$, there exists a constant $c \in(0,1)$, independent of $r$, such that

$$
c \leqslant\left|\frac{\max _{\theta}\left(\varphi_{n}(r \cos \theta, r \sin \theta)+\log r\right)}{\min _{\theta}\left(\varphi_{n}(r \cos \theta, r \sin \theta)+\log r\right)}\right| \leqslant 1 .
$$

Proof. Renormalize the sequence of metrics around $p=\{0\}$ as

$$
\begin{aligned}
& \phi_{n}(z)=\varphi\left(\delta_{n} z\right)+\log \delta_{n}, \\
& \tilde{g}_{n}(z)=e^{2 \phi_{n}(z)}|d z|^{2}=g_{n}\left(\delta_{n} z\right) .
\end{aligned}
$$

Lemma 3.2 yields, for the limit metrics $\varphi$ and $\phi$,

$$
\begin{aligned}
& \lambda^{-1}(\bar{\varphi}(r)+\log r)+C_{3} \leq \varphi(r \cos \theta, r \sin \theta)+\log r \leq \lambda(\bar{\varphi}(r)+\log r)+C_{4}, \\
& \mu^{-1}(\bar{\phi}(r)+\log r)+C_{5} \leq \phi(r \cos \theta, r \sin \theta)+\log r \leq \mu(\bar{\phi}(r)+\log r)+C_{6},
\end{aligned}
$$

with $\lambda, \mu \in(0,1)$. For $r_{1}$ small enough and $n, r_{2}$ big enough, we then have

$$
\begin{aligned}
\lambda^{-1}\left(\bar{\varphi}_{n}\left(r_{1}\right)+\log r_{1}\right)+C_{3} & \leq \varphi_{n}\left(r_{1} \cos \theta, r_{1} \sin \theta\right)+\log r_{1} \\
& \leq \lambda\left(\bar{\varphi}_{n}\left(r_{1}\right)+\log r_{1}\right)+C_{4}, \\
\mu^{-1}\left(\bar{\phi}_{n}\left(r_{2}\right)+\log r_{2}\right)+C_{5} & \leq \phi_{n}\left(r_{2} \cos \theta, r_{2} \sin \theta\right)+\log r_{2} \\
& \leq \mu\left(\bar{\phi}_{n}\left(r_{2}\right)+\log r_{2}\right)+C_{6},
\end{aligned}
$$


where $C_{3}, C_{4}, C_{5}, C_{6}$ are independent of $r$. This last pair of inequalities leads to

$$
\begin{aligned}
\mu^{-1}\left(\bar{\varphi}_{n}\left(r_{2} \delta_{n}\right)+\log \left(r_{2} \delta_{n}\right)+C_{5}\right. & \leq \varphi_{n}\left(r_{2} \delta_{n} \cos \theta, r_{2} \delta_{n} \sin \theta\right)+\log \left(r_{2} \delta_{n}\right) \\
& \leq \mu\left(\bar{\varphi}_{n}\left(r_{2} \delta_{n}\right)+\log \left(r_{2} \delta_{n}\right)\right)+C_{6} .
\end{aligned}
$$

Combining this with (4-1), one obtains, still for $r_{1}$ small enough and $n, r_{2}$ big enough,

$$
\frac{\lambda^{2}}{2} \leqslant\left|\frac{\max _{\theta}\left(\varphi_{n}\left(r_{1} \cos \theta, r_{1} \sin \theta\right)+\log r_{1}\right)}{\min _{\theta}\left(\varphi_{n}\left(r_{1} \cos \theta, r_{1} \sin \theta\right)+\log r_{1}\right)}\right| \leqslant 1
$$

and

$$
\frac{\mu^{2}}{2} \leqslant\left|\frac{\max _{\theta}\left(\varphi_{n}\left(r_{2} \delta_{n} \cos \theta, r_{2} \delta_{n} \sin \theta\right)+\log \left(r_{2} \delta_{n}\right)\right)}{\min _{\theta}\left(\varphi_{n}\left(r_{2} \delta_{n} \cos \theta, r_{2} \delta_{n} \sin \theta\right)+\log \left(r_{2} \delta_{n}\right)\right)}\right| \leqslant 1 .
$$

Thus the max-min inequality holds at the two boundary circles.

Set $u=-\log r=-\log \sqrt{x^{2}+y^{2}}$ and $\theta=\tan ^{-1}(y / x)$. The domain $D \backslash\{0\}$ becomes an infinite annulus $\{(u, \theta): 0<u \leqslant \infty,-\pi \leqslant \theta \leqslant \pi\}$ via this transformation. Let

$$
\xi(u, \theta)=\varphi\left(e^{-u} \cos \theta, e^{-u} \sin \theta\right)-u, \xi_{n}(u, \theta)=\varphi_{n}\left(e^{-u} \cos \theta, e^{-u} \sin \theta\right)-u
$$

and define $\triangle_{u, \theta}=\partial_{u}^{2}+\partial_{\theta}^{2}$. For any small $r_{1}=e^{-u_{0}}>0$, define $\tilde{\varphi}(v, \theta)=\xi\left(v+u_{0}, \theta\right)$ and $\tilde{\varphi}_{n}(v, \theta)=\xi_{n}\left(v+u_{0}, \theta\right)$. Then

$$
\begin{aligned}
& -\triangle_{v, \theta} \tilde{\varphi}=K\left(v+u_{0}, \theta\right) e^{2 \tilde{\varphi}} \quad \text { for all }(v, \theta) \in \tilde{D}, \\
& -\triangle_{v, \theta} \tilde{\varphi}_{n}=K_{n}\left(v+u_{0}, \theta\right) e^{2 \tilde{\varphi}_{n}} \quad \text { for all }(v, \theta) \in \tilde{D} \text {, }
\end{aligned}
$$

where $\tilde{D}=[-1,1] \times S^{1}$. There exists a constant $C$ such that $\tilde{\varphi}, \tilde{\varphi}_{n} \leqslant C$ for $n$ big enough; thus the right-hand sides of both equalities are uniformly bounded in $L^{2}(\tilde{D})$.

Define $\omega, \omega_{n}$ by

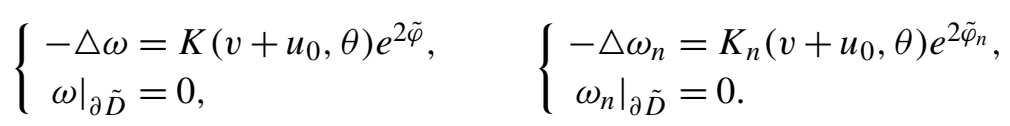

Then $\|\omega\|_{L^{\infty}},\left\|\omega_{n}\right\|_{L^{\infty}}$ are uniformly bounded from above; the bound is actually independent of $u_{0}$, since $L^{2}$ norm of $\tilde{\varphi}(v, \theta)$ and $\tilde{\varphi}_{n}(v, \theta)$ in $\tilde{D}$ converge uniformly to 0 as $n \rightarrow \infty$ and $u \rightarrow \infty$.

The function $h_{n}=\tilde{\varphi}_{n}-\omega_{n}$ is harmonic. Consider the two concentric circles $v_{1}=0\left(|z|=r_{1}\right), v_{2}=\log r_{1}-\log r_{2} \delta_{n}\left(|z|=r_{2} \delta_{n}\right)$. For any circle $v_{3}=\log r_{1}-\log r_{3}$ $\left(|z|=r_{3}\right)$ between the two, set

$$
M_{n}(v)=\max _{v} h_{n}(v, \theta), \quad m_{n}(v)=\min _{v} h_{n}(v, \theta) .
$$


Apply Hadamard's Three-Circle Theorem to obtain

$$
\begin{aligned}
& M_{n}\left(v_{3}\right) \leqslant \\
& \quad \frac{M_{n}\left(v_{1}\right)\left(\log \left(v_{2}+u_{0}\right)-\log \left(v_{3}+u_{0}\right)\right)+M_{n}\left(v_{2}\right)\left(\log \left(v_{3}+u_{0}\right)-\log \left(v_{1}+u_{0}\right)\right)}{\log \left(v_{2}+u_{0}\right)-\log \left(v_{3}+u_{0}\right)}
\end{aligned}
$$

and

$$
\begin{aligned}
& m_{n}\left(v_{3}\right) \geqslant \\
& \quad \frac{m_{n}\left(v_{1}\right)\left(\log \left(v_{2}+u_{0}\right)-\log \left(v_{3}+u_{0}\right)\right)+m_{n}\left(v_{2}\right)\left(\log \left(v_{3}+u_{0}\right)-\log \left(v_{1}+u_{0}\right)\right)}{\log \left(v_{2}+u_{0}\right)-\log \left(v_{3}+u_{0}\right)} .
\end{aligned}
$$

Since the max-min inequality holds at the two boundary circles, we obtain

$$
\begin{aligned}
& M_{n}\left(v_{1}\right)=\max _{\theta} h_{n}(0, \theta)=\max _{\theta}\left(\tilde{\varphi}_{n}(0, \theta)-\omega_{n}(0, \theta)\right) \leqslant \max _{\theta} \tilde{\varphi}_{n}(0, \theta)+C, \\
& m_{n}\left(v_{1}\right)=\min _{\theta} h_{n}(0, \theta)=\min _{\theta}\left(\tilde{\varphi}_{n}(0, \theta)-\omega_{n}(0, \theta)\right) \geqslant \min _{\theta} \tilde{\varphi}_{n}(0, \theta)-C,
\end{aligned}
$$

where $C$ is the uniform bound of $\left\|\omega_{n}\right\|_{L^{\infty}}$. By the definition of $\tilde{\varphi}_{n}(0, \theta)$, we have

$$
\begin{aligned}
\max _{\theta} \tilde{\varphi}_{n}(0, \theta) & =\max _{\theta}\left(\varphi_{n}\left(r_{1} \cos \theta, r_{1} \sin \theta\right)+\log r_{1}\right) \\
& \leqslant \frac{1}{2} \lambda^{2} \min _{\theta}\left(\varphi_{n}\left(r_{1} \cos \theta, r_{1} \sin \theta\right)+\log r_{1}\right)=\frac{1}{2} \lambda^{2} \min _{\theta} \tilde{\varphi}_{n}(0, \theta) .
\end{aligned}
$$

This implies

$$
M_{n}\left(v_{1}\right) \leqslant \frac{1}{2} \lambda^{2} m_{n}\left(v_{1}\right)+2 C .
$$

Similarly, we obtain

$$
M_{n}\left(v_{2}\right) \leqslant \frac{1}{2} \mu^{2} m_{n}\left(v_{2}\right)+2 C .
$$

For any $v_{3} \in\left(v_{1}, v_{2}\right)$, the inequality at the top of this page implies

$$
M_{n}\left(v_{3}\right) \leqslant \frac{1}{2} \min \left(\lambda^{2}, \mu^{2}\right) m_{n}\left(v_{3}\right)+4 C ;
$$

thus

$$
\max _{\theta} \tilde{\varphi}_{n}\left(v_{3}, \theta\right) \leqslant \frac{1}{2} \min \left(\lambda^{2}, \mu^{2}\right) \min _{\theta} \tilde{\varphi}_{n}\left(v_{3}, \theta\right)+6 C
$$

and so

$$
\max _{\theta}\left(\varphi_{n}\left(r_{3}, \theta\right)+\log r_{3}\right) \leqslant \frac{1}{2} \min \left(\lambda^{2}, \mu^{2}\right) \min _{\theta}\left(\varphi_{n}\left(r_{3}, \theta\right)+\log r_{3}\right)+6 C .
$$

Because $\varphi+\log r \rightarrow-\infty$ when $r \rightarrow 0$, and $C$ is independent of $r$, we see that if $r_{1}$ small enough and $n$ big enough, then

$$
\min _{\theta}\left(\varphi_{n}\left(r_{3}, \theta\right)+\log r_{3}\right) \leqslant \max _{\theta}\left(\varphi_{n}\left(r_{3}, \theta\right)+\log r_{3}\right) \leqslant c \min _{\theta}\left(\varphi_{n}\left(r_{3}, \theta\right)+\log r_{3}\right)
$$

for any $r_{3} \in\left(r_{2} \delta_{n}, r_{1}\right)$, where $c \in(0,1)$ is a constant. This immediately implies that the max-min inequality holds in $\operatorname{Neck}\left(r_{1}, r_{2}\right)$. 
Proof of Main Theorem 2.6. Case 1: The renormalized limit metric $\phi$ does not vanish. According to the definition of the weak angle and the out angle, for $r_{1}$ small enough and $r_{2}, n$ large enough we have

$$
\left|\int_{|z|=r_{1}} k_{g_{n}} d s_{n}-\alpha\right|<\varepsilon, \quad\left|\int_{|z|=r_{2} \delta_{n}} k_{g_{n}} d s_{n}-\beta\right|<\varepsilon .
$$

By Lemmas 3.7 and 3.9, we have $\alpha \geqslant 0$ and $\beta \leqslant 0$. If $\alpha>0$, there exists a circle $|z|=r_{3, n}$ such that

$$
\int_{|z|=r_{3, n}} k_{g_{n}} d s_{n}=\frac{\alpha}{2}
$$

and we can assume that $r_{3, n}$ is maximal satisfying this condition. Define

$$
\begin{aligned}
\bar{\varphi}(r) & =\frac{1}{2 \pi} \int_{0}^{2 \pi} \varphi(r \cos \theta, r \sin \theta) d \theta, \\
\bar{\varphi}_{n}(r) & =\frac{1}{2 \pi} \int_{0}^{2 \pi} \varphi_{n}(r \cos \theta, r \sin \theta) d \theta .
\end{aligned}
$$

Then

$$
\begin{aligned}
\iint_{r_{3, n} \leqslant|z| \leqslant r_{1}} \frac{\alpha}{2} e^{2 \bar{\varphi}_{n}} r d r d \theta & \leqslant \frac{1}{2} \int_{0}^{2 \pi} \int_{r_{3, n}}^{r_{1}} e^{2 \bar{\varphi}_{n}} r \int_{|z|=r} k_{g_{n}} d s_{n} d r d \theta \\
& =\pi \int_{r 3, n}^{r_{1}} e^{2 \bar{\varphi}_{n}} r\left(\partial_{r} \bar{\varphi}_{n} r+1\right) d r \\
& =\pi \int_{r 3, n}^{r_{1}} e^{2\left(\bar{\varphi}_{n}+\log r\right)}\left(\partial_{r} \bar{\varphi}_{n}+\frac{1}{r}\right) d r \\
& =\left.\frac{\pi}{2} e^{2\left(\bar{\varphi}_{n}+\log r\right)}\right|_{r_{3, n}} ^{r_{1}} .
\end{aligned}
$$

Using the max-min inequality (Lemma 4.1), one easily gets

$$
\left.e^{2\left(\bar{\varphi}_{n}+\log r\right)}\right|_{r_{1}} \leqslant\left(\frac{1}{2 \pi} \int_{0}^{2 \pi} e^{\left.\left(\varphi_{n}+\log r\right)\right|_{r_{1}}} d \theta\right)^{1 / c},
$$

where $c \in(0,1)$ is the constant in the max-min inequality. Since

$$
\int_{0}^{2 \pi} e^{\left.\left(\varphi_{n}+\log r\right)\right|_{r_{1}}} d \theta<\epsilon
$$

(from the blowing up process), inequalities (4-2) and (4-3) then imply

$$
\iint_{r_{3, n} \leqslant|z| \leqslant r_{1}} \frac{\alpha}{2} e^{2 \bar{\varphi}_{n}} r d r d \theta \rightarrow 0 .
$$


If $\alpha>0$, then

$$
\iint_{r_{3, n} \leqslant|z| \leqslant r_{1}} e^{2 \bar{\varphi}_{n}} r d r d \theta \rightarrow 0
$$

as $r_{1} \rightarrow 0, r_{2} \rightarrow \infty$ and $n \rightarrow \infty$. Using the max-min inequality again, we obtain

$$
\iint_{r_{3, n} \leqslant|z| \leqslant r_{1}} e^{2 \varphi_{n}} r d r d \theta \rightarrow 0 .
$$

On the other hand, for the annulus bounded by two concentric circle $|z|=r_{1}$ and $|z|=r_{3, n}$, we apply the Gauss-Bonnet Theorem to get

$$
\int_{|z|=r_{1}} k_{g_{n}} d s_{n}-\int_{|z|=r_{3, n}} k_{g_{n}} d s_{n}+\int_{r_{3, n} \leqslant|z| \leqslant r_{1}} K_{n} d g_{n}=0
$$

while

$$
\left(\int_{r_{3, n} \leqslant|z| \leqslant r_{1}} K_{n} d g_{n}\right)^{2} \leqslant \int_{r_{3, n} \leqslant|z| \leqslant r_{1}} K_{n}^{2} d g_{n} \int_{r_{3, n} \leqslant|z| \leqslant r_{1}} d g_{n}
$$

and

$$
\int_{r_{3, n} \leqslant|z| \leqslant r_{1}} d g_{n}=\iint_{r_{3, n} \leqslant|z| \leqslant r_{1}} e^{2 \varphi_{n}} r d r d \theta \rightarrow 0 .
$$

This means that $\int_{r_{3, n} \leqslant|z| \leqslant r_{1}} K_{n} d g_{n} \rightarrow 0$. Taking the limit in (4-4), we get $\alpha-\frac{1}{2} \alpha=$ 0 , a contradiction. Thus we have proved that $\alpha=0$ in this case.

Case 2: The renormalized limit metric $\phi_{0}$ vanishes. If the out angle $\beta$ is nonpositive, we apply the Gauss-Bonnet theorem directly:

$$
\int_{|z|=r_{1}} k_{g_{n}} d s_{n}-\int_{|z|=r_{2} \delta_{n}} k_{g_{n}} d s_{n}+\int_{r_{2} \delta_{n} \leqslant|z| \leqslant r_{1}} K_{n} d g_{n}=0 .
$$

Since the limit metric vanishes,

$$
\int_{r_{2} \delta_{n} \leqslant|z| \leqslant r_{1}} K_{n} d g_{n} \rightarrow 0
$$

Taking the limit in the last equality, we have $\alpha-\beta=0$, so $\alpha=\beta=0$.

If $\beta>0$, we consider the bubble tree decomposition. The renormalized limit metric $\phi$ has two bubble points at least, by Theorem 1.2. Assume the bubble points are $\left\{q_{1}, q_{2}, \ldots, q_{m}\right\}$, with $m \geqslant 2$, and the weak angle at $q_{i}$ is $\alpha_{i}$. Let $h_{n}=\tilde{g}_{n}$ be the renormalized metrics, and take disks $D_{r^{(i)}}\left(q_{i}\right)$ around $q_{i}$ with radius $r^{(i)}$ such that

$$
\left|\alpha_{i}-\int_{\partial D_{r^{(i)}}\left(q_{i}\right)} k_{h_{n}} d s_{n}\right|<\varepsilon
$$

for $n$ big enough, and a fixed small $\varepsilon$. Set

$$
\Omega=D_{r_{2}}(p) \backslash \bigcup_{1 \leqslant i \leqslant m} D_{r^{(i)}}\left(q_{i}\right)
$$


(before renormalization, the disk is $D_{r_{2} \delta_{n}}(p)$ ). Now apply to the Gauss-Bonnet Theorem to $\Omega$ :

$$
\int_{|z|=r_{2}} k_{h_{n}} d s_{n}-\sum_{i=1}^{m} \int_{|z|=r^{(i)}} k_{h_{n}} d s_{n}+\int_{\Omega} K_{n} d h_{n}=-(m-1) .
$$

Because $\left\{h_{n}\right\} \rightarrow 0, A_{n}(\Omega)=\int_{\Omega} d h_{n} \rightarrow 0$ when $n \rightarrow \infty$, we have

$$
\left(\int_{\Omega} K_{n} d h_{n}\right)^{2} \leqslant \int_{\Omega} K_{n}^{2} d h_{n} \int_{\Omega} d h_{n} \rightarrow 0
$$

Taking limits in (4-5) we get $\sum_{i=1}^{m} \alpha_{i}=m-1+\beta$. Thus there must exist some $i$ with $1 \leqslant i \leqslant m$ such that $\alpha_{i}>0$. We can assume $\alpha_{1}>0$, and consider a pair of angles $\left\{\alpha_{1}, \beta_{1}\right\}$ (where $\beta_{1}$ is the out angle at the point $q_{1}$ ) and the blowing-up process around $\left\{q_{1}\right\}$. If $\beta_{1} \leqslant 0$, then by the argument above we have $\alpha_{1}=0$, a contradiction. If $\beta_{1}>0$, we argue as in the case $\beta>0$ : we get a new pair of angles $\left\{\alpha_{11}, \beta_{11}\right\}$ at the bubble point $q_{11}$ in the blowing-up process around $q_{1}$, such that $\alpha_{11}>0$. If $\beta_{11} \leqslant 0$, there is a contradiction. If $\beta_{11}>0$, then we apply the blowing-up once more. Since $\beta>0$, the limit metric of renormalization is zero and the limit metric is a ghost vertex in the bubble tree. Thus, if $\alpha>0$, we get an infinite series of ghost vertices in the bubble tree. But we know that there are only finitely ghost vertices in the bubble tree, so we reach a contradiction. This proves that $\alpha$ is zero in this case too, as desired.

\section{References}

[Calabi 1982] E. Calabi, "Extremal Kähler metrics", pp. 259-290 in Seminar on Differential Geometry, Ann. of Math. Stud. 102, Princeton Univ. Press, Princeton, NJ, 1982. MR 83i:53088 Zbl 0487.53057

[Chang and Yang 1988] S.-Y. A. Chang and P. C. Yang, "Conformal deformation of metrics on $S^{2}$ ", J. Differential Geom. 27:2 (1988), 259-296. MR 89b:53079 Zbl 0649.53022

[Chen 1998a] X. Chen, "Extremal Hermitian metrics on Riemannian surfaces", Internat. Math. Res. Notices 15 (1998), 781-797. MR 99i:58035 Zbl 0955.30032

[Chen 1998b] X. Chen, "Weak limits of Riemannian metrics in surfaces with integral curvature bound”, Calc. Var. Partial Differential Eq. 6:3 (1998), 189-226. MR 99k:53087 Zbl 0894.53040

[Chen 1999] X. Chen, "Extremal Hermitian metrics on Riemann surfaces", Calc. Var. Partial Differential Eq. 8:3 (1999), 191-232. MR 2000f:58029 Zbl 0947.53038

[Chen 2001] X. X. Chen, "Calabi flow in Riemann surfaces revisited: a new point of view", Internat. Math. Res. Notices 2001:6 (2001), 275-297. MR 2002c:53111 Zbl 1078.53065

[Chen and Li 1991] W. X. Chen and C. Li, "Classification of solutions of some nonlinear elliptic equations”, Duke Math. J. 63:3 (1991), 615-622. MR 93e:35009 Zbl 0768.35025

[Luo and Tian 1992] F. Luo and G. Tian, "Liouville equation and spherical convex polytopes", Proc. Amer. Math. Soc. 116:4 (1992), 1119-1129. MR 93b:53034 Zbl 0806.53012 
[McOwen 1988] R. C. McOwen, "Point singularities and conformal metrics on Riemann surfaces", Proc. Amer. Math. Soc. 103:1 (1988), 222-224. MR 89m:30089 Zbl 0657.30033

[Troyanov 1991] M. Troyanov, "Prescribing curvature on compact surfaces with conical singularities”, Trans. Amer. Math. Soc. 324:2 (1991), 793-821. MR 91h:53059 Zbl 0724.53023

[Uhlenbeck 1982] K. K. Uhlenbeck, "Connections with $L^{p}$ bounds on curvature", Comm. Math. Phys. 83:1 (1982), 31-42. MR 83e:53035 Zbl 0499.58019

Received December 20, 2005.

\author{
QING CHEN \\ DEPARTMENT OF MATHEMATICS \\ UNIVERSITY OF SCIENCE AND TECHNOLOGY OF CHINA \\ HEFEI, ANHUI, 230026 \\ CHINA \\ qchen@ustc.edu.cn

\section{XIUXIONG CHEN} \\ DEPARTMENT OF MATHEMATICS \\ UNIVERSITY OF WISCONSIN \\ 480 LINCOLN DRIVE \\ MADISON WI 53706-1388 \\ UNITED STATES \\ xxchen@math.wisc.edu \\ WEIYONG HE \\ DEPARTMENT OF MATHEMATICS \\ University of SCIENCE AND TECHNOLOGY OF CHINA \\ HEFEI, ANHUI, 230026 \\ CHINA \\ wyhe@mail.ustc.edu.cn
}

